\title{
Rare diseases treated by plasma proteins
}

\author{
Brian O'Mahony \\ Chief Executive Officer, Irish Haemophilia Society, Cathedral Court, New Street, Dublin 8, Ireland \\ E-mail: brian@haemophilia.ie
}

When plasma is separated or recovered from a blood donation or when plasma is collected by plasmapheresis the end result is not just a fluid which can be used in the treatment of many medical conditions but a raw material which can be used for the production of specific life saving or life enhancing medications for a variety of conditions. These conditions include Haemophilia A and Haemophilia B, von Willebrands Disease a whole range of Primary Immunodeficiencies, Alpha 1 Antitrypsin deficiency, Guillain-Barré Syndrome, Hereditary Angioedema, Idiopathic Thrombocytopenic Purpura and Kawasaki Disease among others. Haemophilia is an inherited bleeding disorder caused by the absence or lower levels of coagulation Factor VIII or Factor IX. Haemophilia is caused by a defect on one of the X-chromosomes and therefore the condition is carried by females and presents almost exclusively in males. Some $30 \%$ of children with haemophilia are spontaneous mutations with no family history. The most common form of Haemophilia is Haemophilia A or Factor VIII deficiency. This occurs in 105 per million males and constitutes approximately $80 \%$ of the cases of Haemophilia. Haemophilia B or factor IX deficiency is less common and occurs in 28 per million males or $20 \%$ of cases. Haemophilia can be classified as severe, moderate or mild. An individual would be classified as having severe Haemophilia if their factor level is less than $1 \%$ of the normal factor level. A person with moderate haemophilia would have $1-5 \%$ of normal factor VIII level and mild would have $5-40 \%$. Severe haemophilia is characterised by spontaneous bleeds into joints and muscles with the initial bleed often occurring when the child is less than 2 years of age. Life threatening bleeds such as bleeds into the central nervous system, intracranial bleeding and bleeding into the throat or internal bleeding can also occur.

Initial treatment for haemophilia in the 1950's and 1960's consisted of whole blood or plasma. In the 1960's Cryoprecipitate was used to treat Factor VIII deficiency and from the 1970's Factor VIII and Factor IX deficiency have been treated with coagulation factor concentrates. Haemophilia A has been treated with Factor VIII concentrates since the early 1970's and haemophilia B has been treated with Prothrombin Complex Concentrates since the 1970's and with Prothrombin Complex Concentrates or specific Factor IX concentrates since the early 1990's. Treatment can be on-demand where bleeding episodes are treated as they occur or prophylactically with Factor VIII three times a week or with Factor IX twice a week. This prevents the bleeds from occurring, minimises joint damage and allows the person 
with Haemophilia to live a relatively normal quality of life. Based on the known incidence of Haemophilia there should be some 400,000 persons with haemophilia world wide. However haemophilia is under diagnosed and under treated especially in many developing or emerging countries. The World Federation of Haemophilia (WFH) in their annual global survey for 2007 [1] identified 142,597 persons with Haemophilia A or B in 105 countries worldwide covering some $89 \%$ of the worlds population. Some 45,000 of these persons with Haemophilia have been identified in 44 European countries.

Von Willebrand Disease (VWD) is the most common type of bleeding disorder. Persons with VWD have a deficiency in a protein known as von Willebrand Factor. VWD is generally less severe than other bleeding disorders such as Haemophilia (with the exception of type 3 VWD). It is estimated that up to $1 \%$ of the world's population may have some form of VWD but the vast majority are asymptomatic. Many persons with VWD may not know that they have the disorder because bleeding symptoms are very mild. There are three types of VWD, type 1, type 2 and type 3 and within each type the disorder can be mild, moderate or severe. Type 1 VWD is the most common form and would generally be mild. Persons with Type 1 VWD have lower than normal levels of von Willebrands Factor. Type 2 VWD involves a defect in the von Willebrands factor structure. The von Willebrand factor protein does not work properly causing lower than normal von Willebrand factor activity. There are different Type 2 von Willebrand disorder defects $(2 \mathrm{~A}, 2 \mathrm{~B}, 2 \mathrm{~N})$. Symptoms are usually moderate. Type 3 VWD is normally the most severe form. People with Type 3 VWD have little or no von Willebrand factor. Symptoms are more severe. People with Type 3 VWD can have bleeding into muscles and joints including spontaneous bleeding similar to haemophilia. Type 1 VWD often does not require treatment or if treatment is required it is generally treated with synthetic Desmopressin (DDAVP). Type 2 VWD can be treated with DDAVP or plasma-derived Factor VIII concentrates which contain sufficient von Willebrand Factor and Type 3 VWD generally requires treatment with plasma-derived factor VIII concentrates which contains von Willebrand factor. VWD can affect both males and females and the vast majority of persons with VWD are not diagnosed especially those with type 1 . Despite the fact that it is the most common bleeding disorder, the WFH have identified only 52,545 persons with VWD globally [1]. Some 25,000 of these are in Europe.

In addition to haemophilia and VWD, there are also a number of rarer factor deficiencies which cause bleeding. These include deficiencies in coagulation Factors I, II, V, VII, X, XI, and XIII. All of these factor deficiencies can occur in both males and females in varying proportions. The WFH have identified 18,762 persons with these rarer bleeding disorders [1]. The most common of these rare bleeding disorders are Factor VII and Factor XI deficiencies. In a country which maintains a comprehensive national register of persons with bleeding disorders it is possible to get a more accurate picture of the relative prevalence of these disorders. In Ireland (May 2009, Data) a national register is maintained at the National Centre for Hereditary 
Coagulation Disorders. This register includes 410 persons with haemophilia A, 203 with haemophilia B, 918 with VWD (381 male and 537 female) and a total of 510 persons with other hereditary bleeding disorders, These include 84 persons with Factor XI deficiency, 55 persons with Factor V deficiency, 46 persons with Factor VII deficiency, 67 persons with Factor X deficiency and 3 persons with Factor XIII deficiency. This also includes some 158 persons with platelet disorders.

Primary Immunodeficiencies (PID) refers to a diverse group of more than one hundred genetic immune disorders many of which result from multi or single defects. The defects may affect one or more components of the immune system and lead to a characteristic increase in susceptibility to recurrent and persistent infections. When Primary Immunodeficiencies are left undiagnosed or are mis-diagnosed the immune system remains compromised often leading to illness, disability, permanent organ damage or even death. PID's are chronic diseases but with early diagnosis and adequate treatment most people with PID can lead a near normal life. The majority of symptomatic and treatable PID's are collectively estimated to have a prevalence of 1 in 10000 and are classified as rare diseases. However experts estimate that 70-90\% of PID's remain undiagnosed and the incidence could be as high as 1 in 500 [2] PID should be suspected in every patient irrespective of age who has recurrent persistent severe or unusual infections. The WHO recognises approximately 70 PIDs including X-linked agammaglobulinaemia (Brutons Disease), Common Variable Immune Deficiency (also called Hypogammaglobulinaemia), Selective IGA Deficiency and Severe Combined Immune Deficiency [3] In undiagnosed or misdiagnosed patients treatment tends to focus on the frequent and long term use of high dose antibiotics. Improved diagnoses of PIDs could therefore have a positive impact on a reduction in the use of antibiotics, better use of health facilities and improvement in the quality of life for the individual. PID's can be diagnosed with a simple and inexpensive blood test which can identify $95 \%$ of these disorders and the treatment generally consists of replacement therapy with plasma derived intravenous immunoglobulins. The International Patients Organisation for Primary Immunodeficiency (IPOPI) estimated that the probable European prevalence of PID's is 60,000 but there are only some 7,000 known patients with PID's [4].

Alpha 1 Antitrypsin Deficiency (AATD) is a genetic condition which causes emphysema of the lungs and cirrhosis of the liver. The only treatment available for liver related Alpha1 Antitrypsin Deficiency is liver transplantation but replacement or augmentation therapy has been developed through a plasma-derived Alpha1 Antitrypsin therapy which can greatly assist in the treatment of persons at risk of emphysema or who have developed emphysema. AATD is greatly under diagnosed. It is estimated by the Alfa Europe that in excess of 100,000 persons on the continent of Europe may have Alpha 1 Antitrypsin Deficiency and yet the majority remain undiagnosed [5]. Many are diagnosed with asthma or chronic obstructive pulmonary disease. This in turn means that they are often inappropriately treated with therapies that do little for their underlying condition. Therapy for AATD is to replace or augment the Alpha 1 Antitrypsin which is lacking. This plasma-derived therapy has been developed and 
is now widely used in the USA and many European countries including Germany, Austria, France, Spain, Ireland, Italy and Portugal. It is not universally available to patients in Europe and in some European countries it is available only on a named patient basis. National patient groups for AATD as well as Alfa Europe are currently promoting detection and screening programs for the condition in many European countries.

Guillain-Barré Syndrome (GBS) is an autoimmune disease affecting the peripheral nervous system usually triggered by an acute infectious process. It is included in the wider group of peripheral neuropathies. There are several types of GBS but the most common is Acute inflammatory demyelinating polyneuropathy (AIDP). It is frequently severe and usually exhibits as an ascending paralysis noted by weakness in the legs which spreads to the upper limbs and the face. Prompt treatment is required with either plasma aphaeresis or high dose intravenous Immunoglobulins. If proper treatment is received the majority of patients will regain full functional capacity. With prompt treatment approximately $80 \%$ of patients have a complete recovery within a few months to a year. About 5-10\% recover with severe disability and the mortality rate is usually about $4 \%$. In addition to AIDP there are five other subtypes of GBS. These are Miller Fisher Syndrome, Acute Motor Axonal Neuropathy, Acute Motor Sensory Neuropathy, Acute Panautonomic Neuropathy and Bickerstaff's Brainstem Encephalitis. The GBS/CIDP support organisation estimated the prevalence of GBS in Europe to be 5,700 [4].

Hereditary Angioedema (HAE) is a rare genetic condition causing episodic angioedema including possibly dangerous laryngeal oedema. Episodes can be unpredictable or triggered by trauma, drugs or dental treatment. HAE affects all races with an estimated prevalence of 1 in 50,000. HAE is caused by a deficiency or a dysfunctional version of the protein $\mathrm{C} 1$ esterase inhibitor. There are three types of HAE. Type 1 has low levels of $\mathrm{C} 1$ esterase inhibitor protein, Type 2 has normal or elevated levels but mutation impairs its function. Type 3 only affects women and is precipitated or worsened by high oestrogen levels. Type 3 is also known as Oestrogen sensitive HAE or variant HAE. For Types 1 and 2 the cause is a defect in the C1 inhibitor gene inheritance and is autosomal dominant. The inheritance pattern with Type 3 is uncertain but is probably dominant. The typical history is recurrent attacks of angioedema and abdominal pain. Most patients have attacks during childhood, although the diagnosis sometimes may not be made until the second or third decade of life. Management of acute severe attacks which can cause life threatening oedema should be carried out by giving treatment with $\mathrm{C} 1$ esterase inhibitor concentrate. If this is not available or in an emergency, fresh frozen plasma or solvent detergent treated plasma can be used. However these may worsen symptoms during the acute phase because they contain complement components.

Idiopathic Thrombocytopenic Purpura (ITP) is a condition where the individual has a low platelet count with no known cause. It is often asymptomatic but a very low platelet count can often lead to symptoms such as purpura or bruising. Incidence of ITP is estimated at 50 to 100 new cases per million of population per year with 
half of those being children. Symptoms include bruising, petechiae, (especially on the extremities) and bleeding from the nostrils and gums. Serious and possibly fatal complications such as intracerebral haemorrhage or lower gastrointestinal bleeding can occur if the platelet count is very low. Diagnosis of ITP is usually a process of exclusion where the clinician has to determine that there are no blood abnormalities other than the low platelet count. Treatment is usually initiated with intravenous steroids or intravenous immunoglobulins or a combination of these therapies. Platelet infusions may be administered in an emergency in order to quickly raise the platelet count. According to the ITP support association the probable prevalence of ITP in Europe is 36,000 with some 3,000 known patients [4].

The variety of conditions treated routinely by plasma-derived therapies is enormous. The degree to which these conditions are generally diagnosed and treated also varies. The vast majority of persons with Haemophilia A and B are diagnosed, the majority of persons with VWD are undiagnosed but it may well be that many of those with Type 3 VWD, which is the most severe, are diagnosed and that many of those who remain undiagnosed may have a milder form of VWD. The majority of persons with PID's and AATD are undiagnosed and therefore untreated. In both cases individuals may be currently treated for the symptoms caused by their primary immune deficiency or their alpha 1 antitrypsin deficiency where a more targeted therapy such as intravenous immunoglobulin or alpha 1 anittrypsin would greatly benefit their survival and quality of life. Treatment for GBS and HAE also varies widely and the availability of an adequate diagnosis and adequate treatment with $\mathrm{C} 1$ esterase inhibitor concentrate or intravenous immunoglobulin can mean the difference between life and death.

The organisations which represent the patients with these conditions work hard to try to ensure that people with these conditions are diagnosed and that appropriate therapy is available to them. In relation to Haemophilia, VWD and the rarer clotting factor defects these patients are represented globally by the World Federation of Hemophilia (WFH) and in Europe by the European Haemophilia Consortium (EHC). The EHC has 44 national member organisations in Europe. Patients with Primary Immunodeficiency are represented globally by the International Patient Organisation for Primary Immunodeficiency (IPOPI) with 27 national European organisations. Patients with Alpha 1 Antitrypsin Deficiency are represented by Alfa Europe with 13 European national organisations. Patients with Guilllain- Barré Syndrome are represented by the GBS/CIDP Foundation. Those with Hereditary Angioedema are represented by HAEI (International patient organisation for $\mathrm{C} 1$ esterase inhibitor deficiencies) and patients with Idiopathic Thrombocytopenic Purpura (ITP) are represented by the ITP Support Association. All of these patients have in common a reliance on therapies manufactured from plasma. although with Haemophilia A and $\mathrm{B}$, recombinant factor concentrates (not manufactured from human plasma) have been available for a number of years as an alternative therapy to plasma-derived concentrates. 
We have seen with many of the conditions including PID's and AATD that they are not diagnosed in the majority of patients. If we look at Haemophilia which is the condition with the highest proportion of patients diagnosed one would expect therefore that therapy would be widely available to all patients in Europe. This is not the case. Access to replacement therapy for Haemophilia varies from no access to regular therapy to on-demand treatment to prophylactic treatment. Replacement therapy used varies from no replacement therapy to plasma to Cryoprecipitate to plasma-derived factor concentrate to recombinant factor concentrates. Due to the relatively high cost of therapy the factor use tends to be related to the economy. The WFH global survey in 2007 [1] demonstrated that in countries with a GNP per capita greater than US $\$ 10,000$, Factor VIII use per capita was 3.47 IU. In those countries where GNP was between $\$ 2,000$ and $\$ 10,000$, Factor VIII use fell to 0.31 IU per capita and in countries with GNP per capita lower than \$2,000 Factor VIII use was $0.02 \mathrm{IU}$ per capita. If we look at Europe in relation to Western, Central and Eastern Europe in the same survey, we find that while the European average was $3.21 \mathrm{IU}$ per capita, in Western Europe the average was 5.99 IU per capita, in Central Europe it was $2.33 \mathrm{IU}$ per capita and in Eastern Europe it was $1.21 \mathrm{IU}$ per capita. In a survey of 13 European countries carried out in January 2009 [6], the per capita Factor VIII use varied from 8.7 IU's per capita in Sweden to 0.38 IU's per capita in Romania - a 23 fold difference in availability of treatment within the European community. Of the 13 countries surveyed only 5 of the countries (Sweden, Ireland, Netherlands, Germany and Hungary) had a per capita Factor VIII use greater than 5 IU per capita, whereas two countries (Romania, Bosnia \& Herzegovina) had a Factor VIII per capita use less than $1 \mathrm{IU}$ per capita. This is below the level which is generally consistent with survival of the person with Haemophilia into adulthood. 10 of the 13 countries surveyed always used plasma-derived factor concentrates.

Despite the fact that many persons with PID's remain to be diagnosed the efficacy of treatment with intravenous Immunglobulin has been recognised and the global demand and use of plasma-derived intravenous immunoglobulin has increased dramatically in recent years. In 2000, some 47.4 metric tonnes of IVIG were used. By 2008 this had increased to 82.32 metric tonnes [7] The continued increase in demand for intravenous immunoglobulin, the continued requirement for plasma-derived Factor VIII and Factor IX concentrates and the increasing diagnosis and therefore requirement for plasma-derived therapies for the other conditions including Alpha 1 Anitrypsin, have lead to an ever increasing requirement for collection of plasma globally. In 2007, it was estimated that some 8.6 million litres of recovered plasma and 17.95 million litres of aphaeresis plasma had been collected to give a total of 26.5 million litres. It is estimated that the global requirement for plasma for fractionation will have reached 41.7 million litres by 2015 , with the majority of this increase being collected in the United States [7]. This forecast for increased requirement does not take into consideration the potential approval of IVIG for the treatment of Alzheimer's disease. If this occurs there will be a further surge in the demand for 
IVIG. Giving the complicated economics of the manufacture of plasma-derived products, this potentially very large surge in demand for the availability of IVIG would be welcome if it leads to a massive increase in supply of IVIG in addition to a similarly massive increase in supply of plasma-derived factor concentrates, alpha 1 antitrypsin and $\mathrm{C} 1$ esterase inhibitor components. This would however require an acceleration in the diagnosis of patients with conditions such as alpha 1 antitrypsin deficiency and willingness by European governments to pay for adequate therapy for all the patients with these rare conditions. It also depends on a continuing and strengthening market for plasma-derived Factor VIII and Factor IX concentrates in a situation where a large number of European countries now rely heavily on the use of recombinant Factor VIII and Factor IX concentrates for routine treatment of haemophilia A and B.

An increasing number of patients diagnosed, together with new indications for IVIG leading to an increased collection of plasma and increased manufacture of plasma derived components is one potential development. It must be a major concern for the patients with PIDs who currently rely on IVIG that if IVIG is indicated for the treatment of Alzheimer's disease in the future, this would potentially lead to an enormous surge in the requirement for IVIG for treatment of Alzheimer's in a situation where the demand for the other components did not also increase to the same extent .The economics behind the production of plasma-derived medicinal products are complex and carefully balanced. In order to maintain a financially viable production, ideally there should be a demand for all proteins for therapeutic purpose contained in plasma. Given this interdependence, a surge in the demand for one protein which is not reflected in the demand for the other proteins would throw off balance the production economics and potentially. patients with PID's and the other conditions reliant on IVIG could be left scrambling for therapy in a competitive situation with Alzheimer's patients. Therefore ensuring adequate diagnosis and working to ensure adequate availability of therapy for these conditions is a major priority for all the organisations in the coming years.

New indications and under diagnosis however are not the only threats to patient access to therapy with plasma-derived components. In the last decade we have seen a number of threats or potential threats to patient access to therapy which have resulted from proposed legislation, ideology and the non application of scientific principles. Following the devastating impact of the infection of thousands of persons with Haemophilia with HIV and Hepatitis C from the 1970's up to the end of the 1980's the European Commission passed into law Directive 89/381/EEC which at the time sought to encourage self sufficiency from voluntary unpaid donors throughout Europe. National self sufficiency in plasma-derived factor concentrates in the 1980's may have led to a lower prevalence of HIV infection in persons with haemophilia in some countries in Europe. The impact of such measures on the infection of people with Haemophilia with Hepatitis $\mathrm{C}$ is hard to characterise as Hepatitis $\mathrm{C}$ has been associated with plasma from every source. However, by the time the Directive was passed in 1989, the concept was already outdated. In 2002, the EU propagated a new directive 2002/98/EC which set minimum standards of quality 
and safety for the collection, testing, processing, storage and distribution of human blood and blood components. During the process of drafting the directive several amendments were tabled which sought to limit or entirely exclude access to plasmaderived therapies which were not manufactured solely from plasma collected in the European Community from voluntary non remunerated blood donors or similarly collected from voluntary non remunerated blood or plasma donors from outside the Community. In the face of the actual reality at the time (the majority of plasma used to manufacture components in Europe was manufactured from the plasma of remunerated plasma donors from within the Community and from the United States) these amendments were not enshrined into law in the directive. The directive did however state that measures should be taken to encourage voluntary non remunerated blood donations in Europe. In 2001, the WFH, the EHC and IPOPI all lobbied to ensure that these well intentioned but erroneous amendments were not enshrined in the directive. If they had been they would have led to a ludicrous situation where the European directive specified a course of action which was neither practical, realistic or necessary. This issue was raised again in early 2008 when the International Federation of Blood Donor Organisations (IFBDO) wrote to the EU Commission in relation to the sufficiency of supply of blood and blood products within the European Union. In their letter they spoke of the need for the EU Commission to "achieve full European self sufficiency with safe blood-plasma derivates from non remunerated donors". They also spoke about "the dumping of cheap products stemming from blood from poor Americans". Again in response to this the WFH, the EHC, IPOPI and Alfa Europe contacted the Commission separately to point out their concerns in relation to this issue. This issue was raised again by EURORDIS, the rare diseases Europe coalition group in a communication to the EU Commission in March 2009. On this occasion EURORDIS expressed their concern about a lack of positive encouragement from the EU for blood and plasma donation (a reasonable point). They expressed concern with regard to the source of plasma imported from the USA implying that, in situations of shortage, some US producers may resort to importing plasma from third countries from regions where inspections are more difficult to perform. These comments are made in the absence of any case studies, examples or any scientific data to lend them credence. The issues relating to the desire to have European self sufficiency for plasma continue to be raised primarily by those who do not rely on the products. The organisations who represent the frequent users of these products such as WFH, EHC, IPOPI, Alpha Europe, GBS/CIDP, HAEI and the ITP Support Association have become increasingly concerned over the past number of years at the number of occasions on which access to vital plasma therapies has been potentially jeopardised by ill informed comments which do not represent either the individual or collective views of these umbrella patient organisations whose members actually use the products

It is perhaps worth bringing some clarity to the whole issue of paid versus unpaid or remunerated versus non remunerated donors. Many people and many organisations would welcome a situation where countries could be self sufficient in blood for 
transfusion. There needs to an understanding of the fundamental differences between blood and plasma; labile blood products and plasma derived medicinal products. Directive 2002/98/EEC applies to blood and plasma as raw material but does not apply to plasma derived medicinal products which are complex, industrially prepared products which have undergone a long and complex production process. Many of the comments would appear to stem from the inappropriate application of past history to current reality, nationalism and an overriding sense of moral and ethical superiority which is contradicted by the science and by the reality of therapy on a global basis. Let us examine the science and the supply reality. In relation to the science, the European Medicines Evaluation Agency (EMEA) have stated that there is no evidence from clinical studies and pharmacovigilance that donor remuneration increases the risk of viral transmission via plasma-derived medicinal products which have been subjected to proper screening at donation and a validated viral inactivation/removal step [8] They also point out that requirements for plasma for fractionation as laid down by the European pharmacopoeia are the same with respect to donor selection and testing of plasma regardless of remuneration of donors. They further state that the safety of plasma-derived medicinal products is ensured by the application of a large number of complementary measures which include inspections of collection and manufacturing facilities, selection of donors, screening of individual donations and testing of pooled plasma units for markers of infection with known viruses and very significantly the application of validated production processes which are capable of inactivating or removing a range of viruses. They state that for plasma aphaeresis donors including those that are remunerated, there are additional voluntary industry standards to ensure that plasma originates from a low risk donor populations. These include qualified donor programs with a high rate of repeat well characterised donors and inventory hold of donations. The greater frequency of donation combined with an inventory hold period increases the probability of detecting an infected donation before its use in the manufacture of medicinal products.

The fact is that plasma derived medicinal products have had an excellent safety record with no transmission of HIV, Hepatitis C or Hepatitis B since the early 1990's. This is true both for components manufactured from the plasma of remunerated and non remunerated donors. Plasma-derived components used in the European Community are subject to the plasma master file requirements of the Community and the plasma pheresis centres are subject to licensing, inspection and regulation by the European Community. The plasma collection facilities used by the industry are also subject to additional voluntary industry standards (the IQPP program) A further measure of safety is conferred by the fact that in a plasma pheresis program the plasma pool for the manufacture of the components required may consist of a smaller number of repeat donors who are frequent attendees and who have been well characterised and tested. Given the past history of infection, the Haemophilia community in particular are very sensitive to the safety of plasma derived products. There is no scientific justification at this point in time for limiting access to plasma from remunerated plasma donors. If such a justification existed the WFH and the 
EHC (as the organisations which represent the collective views of the patients with haemophilia - who use these products on a daily basis - both globally and in Europe respectively) would clearly express their concern. This concern would immediately be expressed also by IPOPI, Alfa Europe and the other organisations.

It is ludicrous in the extreme to try to put in place measures to exclude products manufactured from remunerated plasma donors in a situation where such components constitute $65-70 \%$ of the supply currently used worldwide. Such a shortage would lead to death and the destruction of quality of life for thousands of persons with haemophilia, PID's and other conditions. The view that plasma collected from non remunerated plasma donors in the European Community is somehow safer is palpably not the case when it comes to the risk of vCJD. The United Kingdom, which had a pool of voluntary non remunerated blood donors, can no longer use their plasma for fractionation due to the number of cases of vCJD in the country. There have been three documented transmissions of vCJD through blood transfusion from voluntary unpaid donors in the UK and a number of voluntary unpaid donors in the UK who subsequently developed vCJD donated blood from which the plasma was recovered and used in pools for the manufacture of factor concentrates, IVIG and other medication. We now know [9] that 802 persons with haemophilia have been treated with factor concentrates made from plasma from blood donors in the UK who subsequently developed vCJD and their names are now in an "at risk" register. Similarly because of the high number of BSE, plasma from Ireland is no longer used. Despite the relatively high number of cases of vCJD in France, French plasma from voluntary non remunerated blood donors continues to be used for the manufacture of factor concentrates and IVIG. If one is to object to the use of blood or plasma on safety grounds, it would be more logical currently to object to the continued use of plasma from French non remunerated blood donors than to the use of plasma from remunerated donors from countries such as Germany, Austria and the United States which have not had the same problem with BSE or vCJD. A similar point could be made in relation to plasma from Spain which has also had several cases of vCJD including at least 1 case in a person who had donated blood and plasma.

A separate example of science being subverted by emotion or in this case discrimination issues is seen in the ongoing debate in relation to the continued permanent deferral as blood donors of men who have sex with men (MSM). The MSM donor deferral has been challenged in the USA, Canada, Australia, Netherlands, Ireland and Sweden. This has been challenged in court action in some countries and in the media in other countries. This is generally challenged as unwarranted discrimination and I can understand that such a charge, when levelled against blood collection establishments, leaves them feeling uncomfortable and in a difficult position when trying to explain their policies to groups of potential donors such as university students who, understandably, do not understand the need for discrimination against any group in society. However once again we must and should look to the science. Many groups of people are deferred as donors. They include people who have taken illegal drugs or illegal steroids with a needle even on one occasion, people who have taken money 
or drugs for sex even on one occasion, people who have ever taken clotting factor concentrates, such as persons with haemophilia and people who have resident in the UK for specified periods of time in relation to the risk of vCJD. Interestingly in many countries the spouses or partners of persons with haemophilia are also deferred as donors. Is this discrimination? The answer is yes. Is it warranted? The answer is absolutely yes. In light of the tragic infection of so many thousands of persons with haemophilia with HIV and Hepatitis C in the past, as a community or group of people, persons with haemophilia have a higher prevalence of blood borne viruses and since these blood borne viruses are sexual transmissible it is reasonable and scientifically valid to discriminate against them and against their partners by preventing them from becoming blood or plasma donors. This is also the reason why MSM donors continue to be permanently deferred as donors. A study carried out in Canada [10], estimated the HIV prevalence in the MSM group as approximately $4.2 \%$, compared to $0.016 \%$ in the non-MSM, non-IV drug user male population. If this analysis is correct then an individual donor who truthfully answers yes to the MSM question is 263 times (4.2/0.016) more likely to be HIV infected than a male who truthfully answers no. The exclusion of MSM donors is discriminatory but it is warranted or necessary discrimination. The deferral of MSM donors and of other donors in any category should be reconsidered only when the science predicates reconsideration. The blood establishments should have the fortitude to properly explain the currently applied donor deferral measures and the rationale for these measures to potential donor groups who raise objections or who accuse them of discrimination. It should also be pointed out that no person has a right to give blood but that the patients who rely on blood and plasma products should have a right to get blood or plasma which is safe. Donor deferral and donor testing are carried out primarily for the benefit of the recipient. Let me be clear that blood and plasma donors have rights. They have the right to be treated with respect and dignity, they have the right not to be harmed during the donation process and to be informed of pertinent medical information uncovered through the testing process. They have the right to general information on the use of blood and plasma and indeed many of the patient organisations who represent the users of blood and plasma will gladly speak to donor organisations, when requested, in relation to how there donations are used. They have the right not to be discriminated against without a valid public health reason. They do not however have the right to insist on being a donor contrary to scientific evidence and if there donation is excluded or if they are deferred as donors for a valid scientific reason this is not unwarranted discrimination. Donor selection and deferral most be governed by science and epidemiology.

Issues such as the MSM donor deferral issue or the issue relating to remunerated or non remunerated donors have lead to patient organisations that actually use the products having to make our views known to the EU Commission on a reactive basis on a number of occasions in the recent past. It has indeed been a source of frustration and concern that whereas the views of blood donors are often taken into account by the Commission when drafting directives or guidelines, the views of the persons 
whose very lives and health depend on these vital products have not generally been taking into consideration. In addition, on several of these issues the views of the various patients organisations representing the patients with conditions as diverse as haemophilia, PID, alpha 1 antitrypsin deficiency and Guillian-Barré syndrome have been issued separately by their organisations where in fact there views have coincided entirely.

To this end in March 2008, following an earlier meeting with the EU Commissioner for Health and meetings with representatives from the European Commission's DG SANCO and DG Enterprise a new coalition of plasma users representative organisations (PLUS) was formed. The constituent organisations of PLUS are the EHC, the WFH, IPOPI, The ITP Support Association, HAEI, Alpha Europe and the GBS/CIDP Foundation. Therefore in relation to plasma and plasma related issues, PLUS represents the views of 7 umbrella organisations which in turn represent the views of more than 85 national member organisations within Europe. PLUS represents the views of more than 80,000 known patients and organisations whose members medical conditions have a probable European prevalence in excess of 386,000.

The terms of reference of PLUS are

- To facilitate the exchange of information towards the building of consensus views when possible among the organisations which represent regular users of plasma, plasma proteins and plasma-derived therapies

- To ensure the consensus views of the organisations are communicated to the EU Commission, MEP's, the Council of Europe and other relevant bodies and individuals

- To ensure that the collective views of the organisations are proactively considered on a timely basis when directives, guidelines and recommendations are being framed in relevant areas

- The collective views expressed on behalf of the organisations in no way detract from each organisations ability or right to express their own individual view on any particular issue. PLUS will express their collective view on an issue when agreed.

The organisations that constitute PLUS meet bi-annually and also by conference call twice a year for a total of 4 meetings per year. PLUS is represented externally by a steering group of 3 persons elected by the representative organisations on an annual basis. Discussions took place at a meeting with EU Commissioner Vasilliou in May of 2008 in relation to the formation of PLUS and it was agreed by the Commissioner that the coalition (later designated as PLUS) representing the views of the 7 umbrella organisations would be proactively involved in discussions in relation to future directives and guidelines propagated by the EU including the review of the blood directive and any future review in relation to remuneration or non remuneration of blood and plasma donors. PLUS have now set up an ongoing consultation mechanism with DG Enterprise and DG SANCO where issues relating 
to the concerns of our member organisations in relation to blood and plasma issues will be discussed.

It is no longer acceptable for the views of the thousands of patients who rely on plasma-derived therapies on an ongoing basis to be ignored or not considered when legislation or regulations are being prepared which will affect our access to therapy. Each organisation will have its own position or opinion on any number of issues but when our opinion coincides on fundamental issues in relation to blood or plasma, then our views will be heard collectively through the medium of PLUS. The views of our organisations must no longer be ignored or misappropriated. We are seeking and indeed demanding the right to represent the views of our broad community to ensure that their potential access to life saving or life enhancing therapies will be optimised and enhanced by our collective involvement in the consultation and decision process.

\section{References}

[1] WFH Report on the Annual Global Survey 2007, World Federation of Hemophilia, Montreal.

[2] Prof L. Notoangelo and Prof L. Hammarstrom, presenting to European Parliament Scientific and Technological Assessment Unit, March 2004.

[3] Website of International Patient Organisation for Primary Immunodeficiencies, www.ipopi.org.

[4] O. Mahony B. Rare Plasma Protein Disorders. Presentsation at IPPC Conference, Paris, 2009.

[5] Personal Communication, L. Warren, Alfa Europe.

[6] Haemophilia Treatment in Europe: An Overview, Brian O’Mahony, EHC Conference Warsaw, March 2009.

[7] Global Plasma Demand 2015, Patrick Robert, Market Research Bureau Inc., International Plasma Proteins Congress, Paris, March 2009.

[8] EMEA/CPMP Position statement on remunerated and non remunerated donors, safety and supply of plasma-derived medicinal products. EMEA/CPMP/BWP/ 1812/02/final.

[9] Ministerial Statement to the House of Commons, Westminster, May 2009.

[10] Statement from Canadian Hemophilia society Blood safety and Supply Committee, March 31, 2006. 